\title{
A Comparative Study in Wavelets, Curvelets and Contourlets as Denoising Biomedical Images
}

\author{
Mohamed Ali HAMDI \\ Department of Physics Engineering, Institut National des Sciences Appliquées et de Technologie \\ Ecole Nationale d'Ingénieur de Tunis \\ Tunis, Tunisia \\ E-mail: mohamedalihamdi@yahoo.fr
}

\begin{abstract}
A special member of the emerging family of multi scale geometric transforms is the contourlet transform which was developed in the last few years in an attempt to overcome inherent limitations of traditional multistage representations such as curvelets and wavelets. The biomedical images were denoised using firstly wavelet than curvelets and finally contourlets transform and results are presented in this paper. It has been found that contourlets transform outperforms the curvelets and wavelet transform in terms of signal noise ratio
\end{abstract}

Index Terms - denoising, wavelet, curvelet, contourlet, transform, biomedical image.

\section{INTRODUCTION}

Medical images are usually of low contrast and they are subject to complex type of noise in its acquisition and transmission. All medical images are corrupted by visual noise. The presence of noise gives an image a mottled, grainy, textured, or snowy appearance. This noise can be either image dependent or image independent. It comes from a variety of sources. No imaging modality is free of noise, but noise is much more prevalent in certain types of imaging procedures than in others. Nuclear images are generally the noisiest. Noise is also significant in MRI, Computer Tomography (CT), and ultrasound imaging (US). In comparison to these, radiography produces images with the least noise. Fluoroscopic images are slightly noisier than radiographic images, for reasons explained later. Conventional photography produces relatively noise-free images except where the grain of the film becomes visible.

Recently, a new member of the emerging representation family of multiscale geometric transform is the so-called contourlet transform which was developed in order to overcome limitations of traditional multi-resolution common representation such as curvelets and wavelets. Moreover, the contourlets transform has been efficiently used in medical image denoising [2], and obtained higher quality recovery of edges. In our work, we present a biomedical image" Axial-transverse" denoising using wavelet, curvelet and contourlet transform.

Extrema in gray scale images are often used to crudely extract the image structures. The image is considered as a topographic relief where gray levels correspond to altitude information. Therefore, the dark and light structures of the images correspond to the valleys and the domes of this relief. The regional maxima and minima are respectively the plateaus located at the top of the domes and the bottom of the valleys [9].

The curvelet, like the wavelet transform, is a multiscale transform, with frame elements indexed by location parameters and scale. The curvelet transform was designed to represent singularities and edges along curves much more efficiently than traditional transforms, i.e. using many coefficients for a given accuracy of reconstruction. So, in order to represent an edge to squared error $1 / \mathrm{N}$ requires $1 / \mathrm{N}$ wavelets and only about 1/ $\sqrt{ } \mathrm{N}$ curvelets [15].But contourlet transform is an efficient directional multiresolution expansion that is digital, thus contourlets transform is multiscale, local and directional contour segments and it is constructed via filter banks and can be viewed as an extension of wavelets with directionality than it Inherits the rich wavelet theory and algorithms, it starts with a discrete-domain construction that is amenable to efficient algorithms, and then investigates its convergence to a continuous-domain expansion.[15]. The expansion is defined on rectangular grids i.e. seamless transition between the continuous and discrete worlds.

This paper presents the image de-noising on different Axial-transverse MRI using wavelet transform, curvelet transform and contourlet transform. The performances of the three transforms are compared in terms of Peak Signal to Noise Ratio (PSNR) and the results are presented. 


\section{THE CURVELET TRANSFORM}

The Curvelet transform CT is a multiscale directional transform and a higher dimensional of the Wavelet transform which allows an optimal non adaptive representation of edges designed to represent images at different scales and different angles. [3][16] Curvelet has two mathematical properties [15][17]:

The curvelet frame [15] $\left\{\varphi_{0}\right\}$ is the wave packet frame on $\mathbb{L}^{2} \mathbb{R}^{2}$ based on the second dyadic decomposition:

$$
f(x)=\Sigma_{F} C_{Y} \varphi_{Y}(x) \quad \text { et } C_{Y}=\int f(x) \varphi_{Y}(x) d x \text {; }
$$
after the Frequency shells is $2^{k}<|\xi|<2^{k+1}$; and the Angular sectors is $\propto\left(\omega_{0}, \xi\right) \leq 2^{-k / 2}$ therefore the approximation rate is optimal: Firstly we choose $n$ largest coefficients $C_{p}$ in $f=\sum C_{p} \rho_{p}$ after $\left\|\mathrm{f}-\mathrm{f}_{\mathrm{n}}\right\|_{\mathbb{L}^{2}}^{2} \leq \mathrm{n}^{-2} \log (\mathrm{n})^{\mathbb{2}}$, yet no frame can do better for jumps along curves, finally the wavelet expansion is $\left\|\mathrm{f}-\mathrm{f}_{\mathrm{n}}\right\|_{\mathrm{L}^{2}}^{2} \leq \mathrm{n}^{-1}$.
- Curved singularities should be approximated with some coefficients and in a non-adaptive representation so its name «curvelets."

- The Curvelet transform remain coherent waveforms under an action of a wave equation in the smooth medium
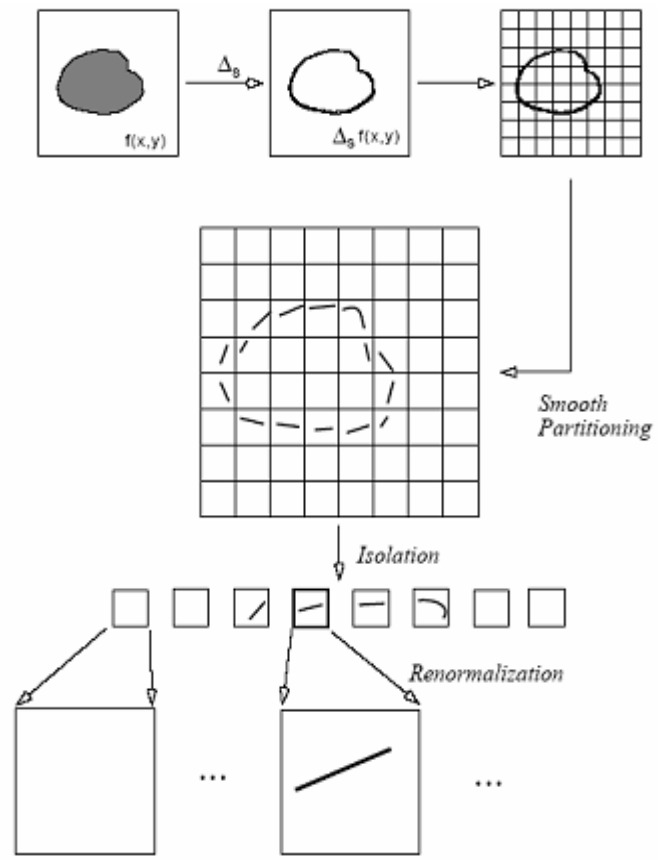

Figure 1. Curvelet Spatial decomposition

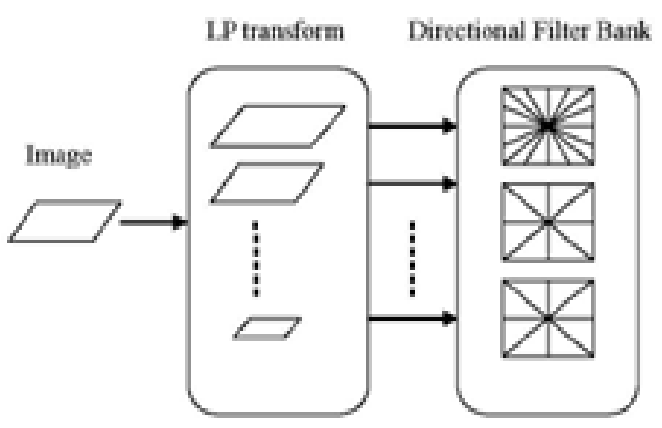

Figure 2. The Contourlet transform framework [8]

This figure shows a flow diagram of the contourlet transform 


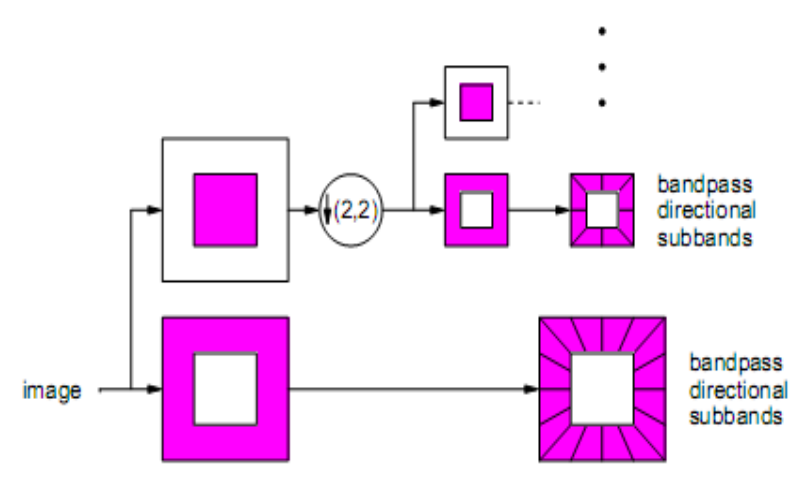

Figure 3. The Contourlet filter bank [6].

This figure shows the contourlet filter bank. Firstly, multi scale decomposition by the Laplacian pyramid [12] [13], then we applied a directional filter bank to each band pass channel. The method used is:

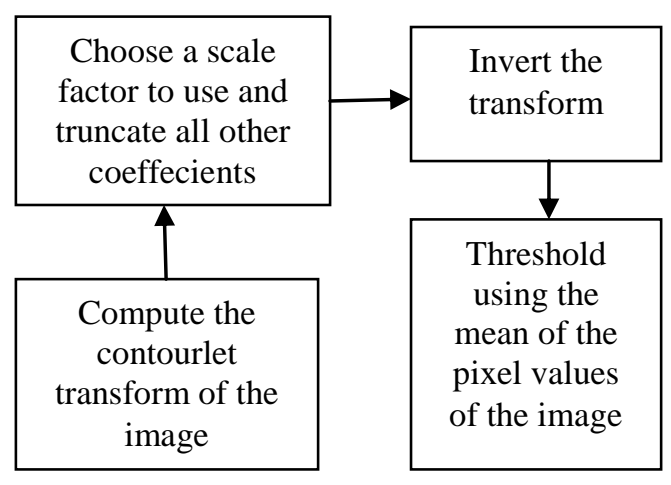

Figure 4. The directional filter bank

\section{THE WAVELET TRANSFORM}

A wavelet is a localization function of the mean equal zero. Wavelet transforms always incorporate a result in a pyramidal representation. There are many cases where a set of successively smoother versions of an image are not down sampled. Wavelet transform is computationally efficient, and part of the reason for this is that the scaling or wavelet function used is often of compact support, i.e. defined on a finite and limited domain.

Wavelets also usually allow an exact reconstitution of original data. A sufficient condition for this in the continuous case wavelet transform is the wavelet coefficients and which allow reconstitution, are of zero mean. Wavelet functions are often wave like but clipped to a finite domain, that's why they are so named. In the discrete case, the wavelet function is sampled at discrete mesh points using, [15] not Dirac sampling distributions, but rather a smoothing function, $\varphi$. Inner products with the wavelets can be performed in the original domain or in the Fourier domain; the former is preferred when the support of the wavelet function is small i.e., it is nonzero on a limited number of grid points. The discrete Wavelet Transform (DWT) can be derived from this theorem if the signal under consideration is band limited. If we consider images, we can't that the frequency band is always limited by the size of the Aperture's camera. The DWT algorithm can be extended to other dimension by separable products of a scaling function $\varphi$ and a wavelet $\psi$. For instance, the 2 dimensional algorithms are based on separate variables leading to prioritizing of vertical, horizontal and diagonal directions. The scaling function is defined by $\varphi(t 1, t 2)=\varphi(t 1) \varphi(t 2)$, and the passage from one resolution to the next is achieved by:

$C_{j+1}\left[k_{s} l\right]=\sum_{m, n} h[m-2 k] h[n-2 l] C_{j}[m, n]=\left[h h * C_{j}\right]_{222}[k, l]$

(1)

We used the DWT algorithm:

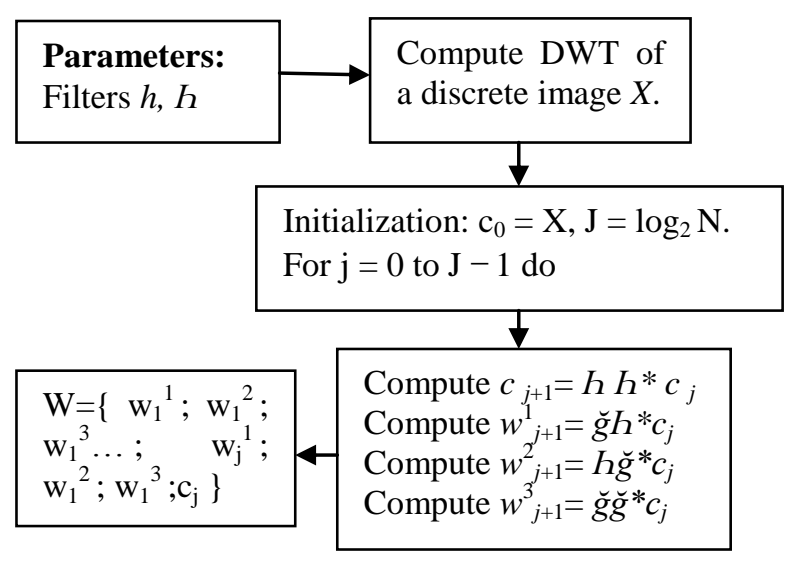

\section{MATERIALS AND METHODS}

Neural Axial-transverse MRI of ten patients were denoised using wavelet curvelet and contourlet transforms. Various types of noise like the Random noise, Gaussian noise, Salt, Pepper and speckle noise were added to this image.

$>$ A multiplicative noise factor of 0.05 is used in case of speckle noise.

$>$ In case of Gaussian white noise, the mean is 0 and variance is 0.05 .

$>$ A Noise factor of 25 is used for random noise.

$>$ The noise density used in case of salt and pepper noise is 0.04 .

The PSNR is the most commonly used as a measure of quality of reconstruction in image denoising[10]. The PSNR for both noisy and denoised images were identified using the following formula:

$$
\text { MSE }=\frac{1}{m} \sum_{i=0}^{m-1} \sum_{j=1}^{n-1}\left\|I(i, j)-K\left(i_{j} j\right)\right\|^{2}
$$

Mean Square Error (MSE) which requires two $\mathrm{m} \times \mathrm{n}$ grey-scale images $\mathrm{I}$ and $\mathrm{K}$ where one of the images is considered as a noisy approximation of the other is defined as [11]:

The PSNR is defined as:

$$
\text { PSNR }=10 \log _{10}\left(\frac{W A X_{T}^{2}}{\text { WISE }}\right)=20 \log _{10}\left(\frac{M A X_{S}}{\sqrt{\text { MYSE }}}\right)
$$

Here, MAXI is the maximum pixel value of the image. The PSNR of the images denoised is compared using wavelet, curvelet and contourlet transform for each type of noise mentioned above. Then the mean and standard deviation of each noise was calculated. 


\section{RESULTS AND DISCUSSION}

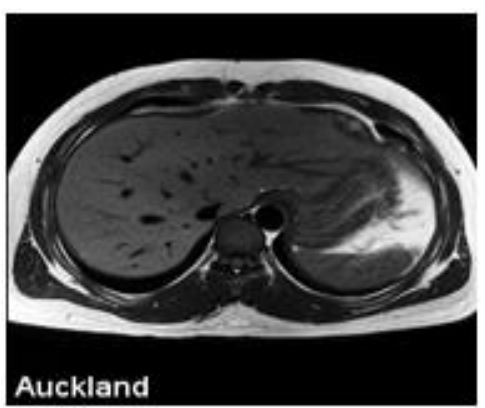

Figure 5. The original image

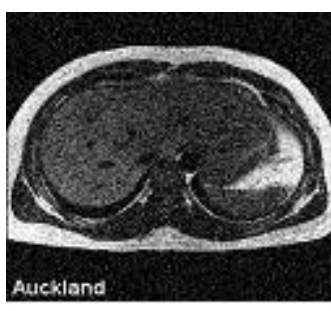

Hoisy inage (Rndom roise)

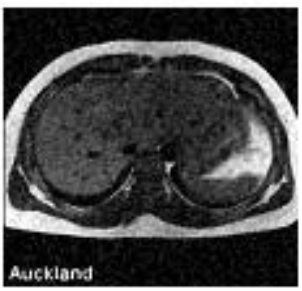

Deroisedimaze (Curoelet) $\mathrm{PSHR}=752$

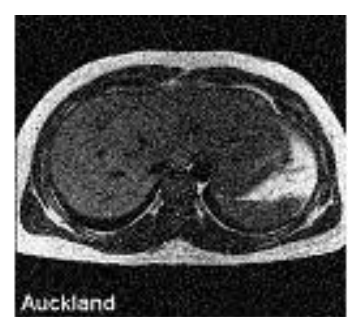

Deroised innge( Whelet) $\mathrm{PSHR}=1182$

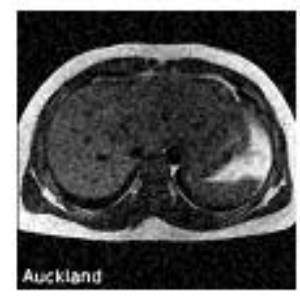

Deroised inse ('Contarlet) PSHR $=7.42$
Figure 6. Denoising of an axial-transverse image of patient 1 with Random noise.
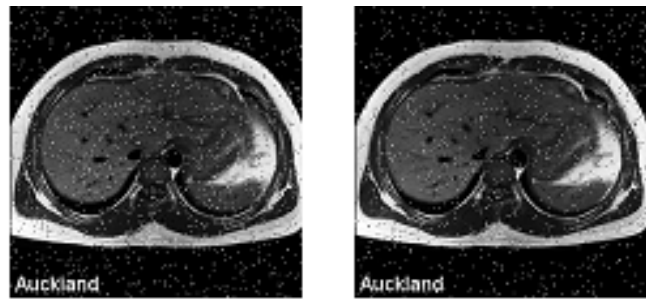

Woisyimage ('Sal \& Perper roise) Deroised imaze(Contarlet)

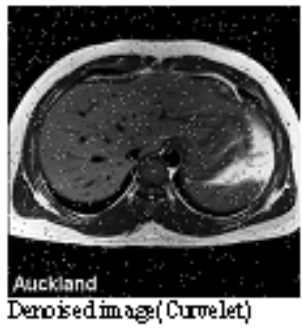

PSKR $=1031$

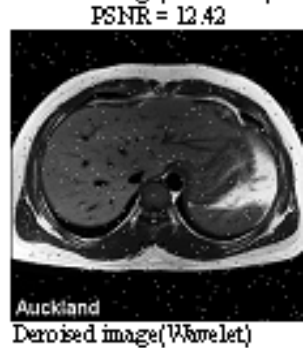

PSHR $=9.12$

Figure 7. Denoising of an axial-transverse image of patient 1 with Salt and Pepper noise
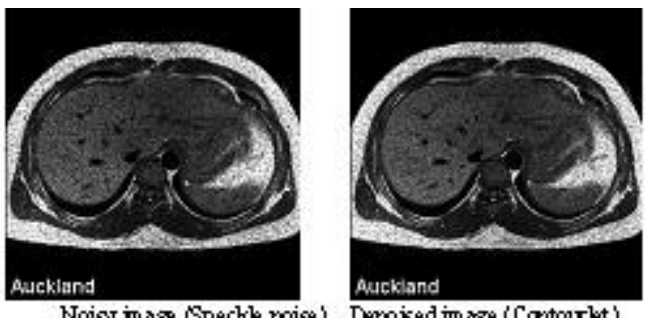

Hoisy inge (Spechle roise) Deroisedimage (Cantorrlt)

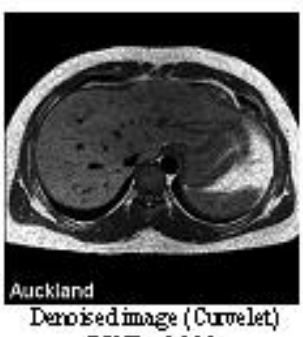
PSHR $=1632$

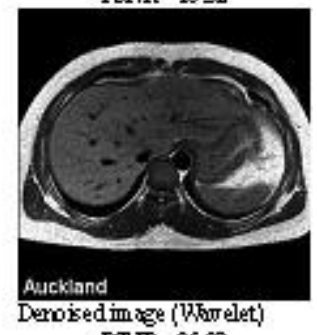

FSHR $=2523$

Figure 8. Denoising of an axial-transverse image of patient 1 with Speckle noise

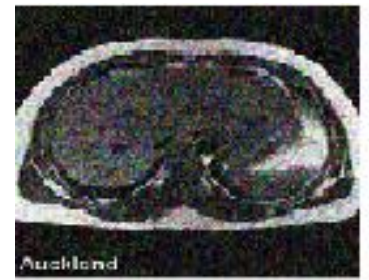

Hoisy image ('Guxsimnoise')

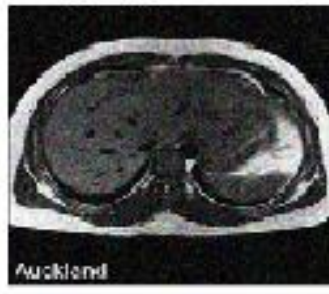

Denoisedimage ('Curvelat) $\mathrm{PSHR}=12.13$

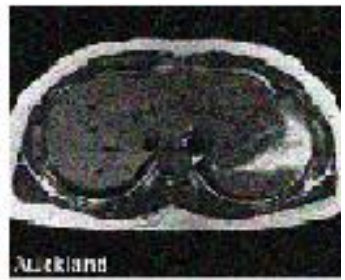

Denoisedinage (Whelet) PSHR $=19.42$

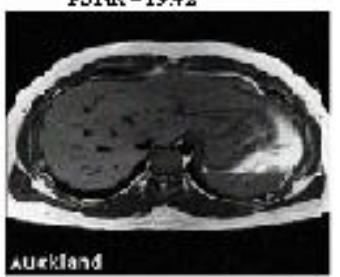

Deroised im ase (Cortarlet) PSKR $=7.32$

Figure 9. Denoising of an axial-transverse image of patient 1 with Gaussian noise 
TABLE I. PSNR VALUES FOR DENOISED AXIAL TRANSVERSE IMAGE

\begin{tabular}{|c|c|c|c|c|}
\hline Patient & Noise & $\begin{array}{l}\text { Curvelet } \\
\text { denoised image } \\
\operatorname{PSNR}(\mathrm{dB})\end{array}$ & $\begin{array}{l}\text { Wavelet } \\
\text { denoised image } \\
\text { PSNR }(\mathrm{dB})\end{array}$ & $\begin{array}{l}\text { Contourlet } \\
\text { denoised image } \\
\text { PSNR }(\mathrm{dB})\end{array}$ \\
\hline \multirow{4}{*}{1} & Random noise & 7.52 & 11.82 & 7.42 \\
\hline & Gaussian noise & 12.13 & 19.42 & 7.32 \\
\hline & Salt \& Pepper noise & 10.31 & 9.12 & 12.42 \\
\hline & Speckle noise & 15.28 & 25.23 & 16.32 \\
\hline \multirow{4}{*}{2} & Random noise & 6.23 & 10.22 & 12.32 \\
\hline & Gaussian noise & 12.11 & 9.25 & 13.32 \\
\hline & Salt \& Pepper noise & 13.36 & 13.26 & 15.02 \\
\hline & Speckle noise & 18.26 & 17.5 & 20.34 \\
\hline \multirow{4}{*}{3} & Random noise & 7.23 & 11.32 & 14.56 \\
\hline & Gaussian noise & 13.23 & 10.65 & 20.32 \\
\hline & Salt \& Pepper noise & 14.25 & 15.22 & 22.6 \\
\hline & Speckle noise & 15.98 & 19.32 & 19.26 \\
\hline \multirow{4}{*}{4} & Random noise & 9.48 & 13.69 & 21.74 \\
\hline & Gaussian noise & 9.32 & 12.36 & 19.65 \\
\hline & Salt \& Pepper noise & 10.69 & 14.23 & 18.33 \\
\hline & Speckle noise & 15.98 & 18.23 & 22.38 \\
\hline \multirow{4}{*}{5} & Random noise & 10.32 & 14.56 & 22.33 \\
\hline & Gaussian noise & 8.65 & 13.65 & 21.97 \\
\hline & Salt \& Pepper noise & 8.14 & 15.23 & 19.33 \\
\hline & Speckle noise & 16.32 & 18.27 & 21.12 \\
\hline \multirow{4}{*}{6} & Random noise & 8.32 & 15.47 & 21.23 \\
\hline & Gaussian noise & 6.25 & 11.23 & 16.23 \\
\hline & Salt \& Pepper noise & 12.96 & 15.32 & 11.25 \\
\hline & Speckle noise & 13.02 & 14.39 & 19.83 \\
\hline \multirow{4}{*}{7} & Random noise & 16.71 & 18.19 & 15.17 \\
\hline & Gaussian noise & 9.72 & 11.13 & 21.96 \\
\hline & Salt \& Pepper noise & 12.65 & 18.17 & 20.39 \\
\hline & Speckle noise & 12.10 & 15.37 & 18.93 \\
\hline \multirow{4}{*}{8} & Random noise & 6.87 & 11.32 & 16.97 \\
\hline & Gaussian noise & 11.97 & 19.23 & 21.87 \\
\hline & Salt \& Pepper noise & 18.13 & 16.32 & 15.98 \\
\hline & Speckle noise & 12.45 & 17.37 & 21.65 \\
\hline \multirow{4}{*}{9} & Random noise & 16.75 & 14.75 & 17.98 \\
\hline & Gaussian noise & 19.32 & 14.27 & 18.19 \\
\hline & Salt \& Pepper noise & 19.32 & 11.23 & 16.34 \\
\hline & Speckle noise & 19.32 & 12.65 & 22.65 \\
\hline \multirow{4}{*}{10} & Random noise & 17.23 & 8.65 & 19.54 \\
\hline & Gaussian noise & 19.54 & 11.23 & 19.23 \\
\hline & Salt \& Pepper noise & 16.87 & 6.32 & 21.32 \\
\hline & Speckle noise & 14.56 & 12.34 & 18.98 \\
\hline
\end{tabular}


We compared the result images after applying wavelet, Curvelet and Contourlet transform to MRI images. It can be observed that region in the result image of Contourlet transform is restored smoother than wavelet and Curvelet transform. The visual quality of various directional texture region of MRI image with Contourlet is also as clear as Curvelet. And the texture region of the MRI image is evident as in result image of wavelet transform. These observation results show that the Contourlet transform obtains better visual quality result image than wavelet transform and Curvelet transform.

Peak signal noise ratio (PSNR) is often employed to compare and measure the difference between result image and original image. It can be observed that the Contourlet transform obtains the highest PSNR value. So the Contourlet approach improves the PSNR value while obtaining high visual quality result images

\section{CONCLUSION}

In the all cases it was found that the Contourlet transform outperforms the Curvelet and Wavelet transform in terms of PSNR and the Contourlet denoised images appear visually more pleasant than the curvelet and the wavelet denoised images. The Contourlet transform provides high PSNR values and can remove the Gaussian white and Random noises from biomedical images very efficiently than the curvelet and the wavelet transform. The Contourlet transform doesn't effectively remove the Speckle noise and Salt and Pepper noise from the biomedical images, and so Contourlet transform is not suited for removal of these two noises though it recovers the edges perfectly

\section{ACKNOWLEDGMENT}

We thank anonymous referees for their constructive comments.

\section{REFERENCES}

[1] Do and Vetterli, "The Contourlet Transform: An efficient Directional Multi Resolution Image Representation", IEEE Transactions on Image Processing, Vol. 14, pp: 2091-2106, 2005.

[2] Effectiveness of Contourlet vs Wavelet Transform on Medical Image Compression: a Comparative Study Negar Riazifar, and Mehran Yazdi World Academy of Science, Engineering and Technology 492009.

[3] Donoho D. L. and Duncan M. R. 1999. Digital Curvelet Transform Strategy, Implementation and Experiments. http://www.curvelet.org/papers/DCvT99.pdf.

[4] Liu Gang; Luo Xutao; Liang Xiaogeng; Fan Bo "An Adaptive Denoising and Enhancing Algorithm Based on the MAP Rule in the Contourlet Domain for Infrared Image "IEEE CONFERENCES, Computational Intelligence and Software Engineering, 2009. CiSE 2009. International Conference on Digital Object Identifier: 10.1109/CISE.2009.5366544 Publication Year: 2009, Page(s): $1-5$
[5] Liu, Li-Wei; Chen, Xin-Wu; Ying, Zhi-Wei; "Texture image retrieval algorithm with dual tree complex contourlet and three statistical features " IEEE CONFERENCES Communication Software and Networks (ICCSN), 2011 IEEE 3rd International Conference on Digital Object Identifier: 10.1109/ICCSN.2011.6013609 Publication Year: 2011 , Page(s): 349 - 352

[6] Masood, H.; Asim, M.; Mumtaz, M.; Bin Mansoor, A.; "Combined Contourlet and Non-subsampled Contourlet Transforms Based Approach for Personal Identification Using Palmprint "IEEE CONFERENCES, Digital Image Computing: Techniques and Applications, 2009. DICTA '09. Digital Object Identifier: 10.1109/DICTA.2009.73 Publication Year: 2009 , Page(s): 408 - 415

[7] ] Xin-Wu Chen; Xiang-Ying Li; Jian-Zhong Ma;" Contourlet-1.3 and Generalized Gaussian model texture image retrieval ",IEEE CONFERENCES, Educational and Information Technology (ICEIT), 2010 International Conference on Volume: 1 Digital Object Identifier: 10.1109/ICEIT.2010.5607640 Publication Year: 2010 , Page(s): V1-458 - V1-462

[8] Zhiling Long; Younan, N.H.; "Contourlet Image Modeling with Contextual Hidden Markov Models"IEEE CONFERENCES, Image Analysis and Interpretation, 2006 IEEE Southwest Symposium on Digital Object Identifier: 10.1109/SSIAI.2006.1633745 Publication Year: 2006, Page(s): 173 - 177

[9] Qu Xiaobo; Yan Jingwen; "The cycle spinning-based sharp frequency localized contourlet transform for image denoising"Intelligent System and Knowledge Engineering, 2008. ISKE 2008. 3rd International Conference on Volume: 1 Digital Object Identifier: 10.1109/ISKE.2008.4731121 Publication Year: 2008 , Page(s): $1247-1251$

[10] Al-Azzawi, N.A.; Mat Sakim, H.A.; Wan Abdullah, A.K.;" An efficient medical image fusion method using contourlet transform based on PCM" Industrial Electronics \& Applications, 2009. ISIEA 2009. IEEE Symposium on Volume: 1, Digital Object Identifier: 10.1109/ISIEA.2009.5356493

Publication Year: 2009, Page(s): $11-14$.

[11] Sveinsson, J.R.; Benediktsson, J.A.; "Combined Wavelet and Contourlet Denoising of SAR Images" Geoscience and Remote Sensing Symposium, 2008. IGARSS 2008. IEEE International Volume: 3 Digital Object Identifier: 10.1109/IGARSS.2008.4779559 Publication Year: 2008 , Page(s): III - 1150 - III - 1153.

[12] Zuofeng Zhou; Jianzhong Cao; Weihua Liu;" Contourlet-based image denoising algorithm using adaptive windows" IEEE CONFERENCES Industrial Electronics and Applications, 2009. ICIEA 2009. 4th IEEE Conference on Digital Object Identifier: 10.1109/ICIEA.2009.5138888 Publication Year: 2009, Page(s): 3654 - 3657

[13] Shun-feng Ma; Geng-feng Zheng; Long-xu Jin; Shuang-li Han; Ran-feng Zhang; "Directional multiscale edge detection using the contourlet transform" Advanced Computer Control (ICACC), 2010 2nd International Conference on Volume: 2 Digital Object Identifier: 10.1109/ICACC.2010.5487180 Publication Year: 2010 , Page(s): $58-62$

[14] Hoang Ng-Duc; Thuong Le-Tien; Tuan Do-Hong; Cao Bui-Thu; Ty Ng-Xuan;" Image retrieval using Contourlet based interest points" Information Sciences Signal Processing and their Applications (ISSPA), 2010 10th International Conference on Digital Object Identifier: 10.1109/ISSPA.2010.5605500 Publication Year: 2010 , Page(s): $93-96$

[15] Jean-Luc Starck, Fionn Murtagh, Jalal M. Fadili, "SPARSE IMAGE AND SIGNAL PROCESSING Wavelets, Curvelets, Morphological Diversity“ cambridge university press Cambridge, New York, Melbourne, Madrid, Cape Town, Singapore, S ao Paulo, Delhi, Dubai, Tokyo Cambridge University Press 32 Avenue of the Americas, New York, NY 10013-2473, USA www.cambridge.org Information on this title: www.cambridge.org/9780521119139 
[16] Starck J.L., Candes E. and Donoho D.L. 2002. The Curvelet Transform for Image Denoising. IEEE Transactions on Image Processing. Vol. 11(6): 670-684.

[17] Starck J.L., Candes E., Murtagh F. and Donoho D.L. 2003. Gray and Color Image Contrast Enhancement by the Curvelet Transform. IEEE Transaction on Image Processing. Vol. 12(6): 706-717.

[18] Fodor, I. K. and C. Kamath. 2003. Denoising through Wavelet Shrinkage: An Empirical Study. SPIE Journal on Electronic Imaging. Vol. 12(1): 151-160.

Mohamed Ali HAMDI was born in Tunisia, received the Ph.D. degree in image processing, from the National University of engineering of TUNIS; He is Assistant Professor of Applied and Computational Mathematics and electronic, National institute of applied sciences and technology. His research interests are in the areas of digital signal processing (DSP), statistical estimation and their applications to signal and image processing and scientific computing. 